Research Paper

\title{
Cancer-Associated Fibroblasts Correlate with Tumor-Associated Macrophages Infiltration and Lymphatic Metastasis in Triple Negative Breast Cancer Patients
}

Jing Zhou ${ }^{1,7^{*}}$, Xiao-Hua Wang ${ }^{2 *}$, Yi-Xin Zhao ${ }^{3^{*}}$, Cheng Chen ${ }^{4}$, Xin-Yun Xu ${ }^{5}$, Qi sun ${ }^{5}$, Hong-Yan Wu ${ }^{5}$, Ming Chen ${ }^{5}$, Jian-Feng Sang ${ }^{1}$, Lei Su ${ }^{1}$, Xiao-Qiao Tang ${ }^{1}$, Xian-Biao Shi ${ }^{1}$, Yin Zhang, ${ }^{1}$ Qiao Yu ${ }^{6}$, Yong-Zhong Yao ${ }^{1}$ $3 \bowtie$, Wei-Jie Zhang $1^{\bowtie}$

1. Dept. of General Surgery, Affiliated Drum Tower Hospital of Nanjing University Medical School, Nanjing, China;

2. Dept. of Medical Oncology, Jiangsu Cancer Hospital Affiliated to Nanjing Medical University, Nanjing, China;

3. School of Medicine, Southeast University;

4. Dept. of Radiotherapy, Jiangsu Cancer Hospital Affiliated to Nanjing Medical University, Nanjing, China;

5. Dept. of Pathology, Affiliated Drum tower Hospital of Nanjing University Medical School, Nanjing, China;

6. Dept. of Breast Surgery, Jiangsu Cancer Hospital Affiliated to Nanjing Medical University, Nanjing, China;

7. Dept. of General Surgery, Kunshan Fourth People's Hospital, Suzhou, China

* Jing Zhou, Xiao-Hua Wang and Yi-Xin Zhao equally contributed to this study.

$\bowtie$ Corresponding authors: Wei-Jie Zhang, E-mail:zhangweijie1616@163.com; Yong-Zhong Yao, E-mail: loyal1006@hotmail.com

(c) Ivyspring International Publisher. This is an open access article distributed under the terms of the Creative Commons Attribution (CC BY-NC) license (https://creativecommons.org/licenses/by-nc/4.0/). See http://ivyspring.com/terms for full terms and conditions.

Received: 2018.07.17; Accepted: 2018.09.22; Published: 2018.11.24

\begin{abstract}
Background: Cancer-associated fibroblasts (CAFs) have been shown to be among the most prominent cells in tumor microenvironment and play a significant role in accelerating tumor metastasis by interacting with other type of cells. Tumor-associated macrophages (TAMs), the predominant tumor-infiltrating immune cells, also play important roles in cancer progression. Here, we aimed to evaluate the effects of CAFs on infiltration of TAMs and lymphatic metastasis in triple-negative breast cancer (TNBC).

Material and methods: The study included 278 patients with histologically confirmed TNBC. Immunohistochemical staining of $\alpha$-smooth muscle actin and fibroblast activation protein were used to identify CAFs. Polarized functional status of infiltrated TAMs was detected by expression of CD163. The clinicopathological features were assessed from all the patients' medical records.

Results: The CAFs-related markers were found to be expressed more frequently in TNBC patents with aggressive behaviors, including recurrence and poor histological differentiation. High activation of CAFs was positively correlated with elevated infiltration of polarized CD163-positive TAMs and lymph node metastasis in TNBC patients. Multivariate Cox analysis revealed that the activation of CAFs, TAMs infiltration, and lymph node metastasis were independent prognostic factors for disease-free survival in TNBC patients.
\end{abstract}

Conclusion: Cancer-associated fibroblasts were associated with infiltration of CD163-positive macrophages and lymphatic metastasis, and may be potential prognostic predictors of TNBC.

Key words: triple-negative breast cancer, cancer-associated fibroblasts, tumor-associated macrophages, lymphatic metastasis, prognosis

\section{Introduction}

Breast cancer is by far the most common cause of cancer-related death for women in China [1]. Based on

the expression of estrogen receptor (ER), progesterone receptor (PR) and human epidermal growth factor 
receptor-2 (HER-2), breast cancers can be categorized into five different molecular subtypes and each subtype has its own characteristics in the clinicopathological features, therapeutic methods and prognosis. Triple-negative breast cancer (TNBC), which is denoted by negative expression of ER, PR and HER2, constitute a heterogeneous group of breast cancers that largely coincide with the basal-like subtype $[2,3]$. With heterogeneity and uncharacterized molecular pathways, TNBC has attracted more attention both clinically and experimentally because of its highly metastatic potential, poor prognosis and insensitive to hormonal or targeted therapy [4-6].

The aggressive behavior of TNBC cells is closely dependent on the tumor microenvironment. The fibroblasts, known as cancer-associated fibroblasts (CAFs), are the predominant stromal cell type in tumor microenvironment. CAFs are distinct from fibroblasts in their expressions of alpha-smooth muscle actin (a-SMA) and fibroblast activation protein (FAP), and are known to play a critical role in tumor growth, angiogenesis, and treatment resistance $[7,8]$. Several studies demonstrated that high activation of fibroblasts was associated with poor outcome in various tumors $[8,9]$.

Aside from CAFs, macrophages are also abundant in the tumor stroma at all stages of tumor progression and are known as tumor-associated macrophages (TAMs). Clinicopathological studies have demonstrated that high infiltration of CD163positive TAMs is associated with poor prognosis in various cancers, including patients with TNBC $[10,11]$.

Accumulating researches have shown that CAFs and TAMs are synergistically related with cancer progression and prognosis of patients [12-16]. However, the precise mechanisms driving the interaction and cross-talk between these cells remain unclarified. We speculated that CAFs might induce TAMs infiltration and consequently promote TNBC metastasis. In our study, we examined the correlation of CAFs and TAMs in TNBC, and analyzed their associations with clinicopathological features including lymph node metastasis and prognosis.

\section{Materials and methods}

\section{Patients and Specimens}

This study included 278 female TNBC patients (the lack of ER, PR and HER2 expression) without evidence of distant metastasis at the time of operation. Tissue samples were obtained from Nanjing Drum Tower Hospital and Jiangsu Cancer Hospital. All specimens were pathologically reassessed independently by two breast pathologists according to the 7th edition of the American Joint Committee on Cancer (AJCC) of Breast Cancer [17]. None of these 278 patients received neoadjuvant chemotherapy or radiation therapy before operation.

\section{Ethics statement}

This retrospective study protocol was approved by the clinical research ethics committee of both hospitals, and complied with the Declaration of Helsinki. Informed consent was obtained from all participants or relatives.

\section{Immunohistochemical Staining}

All the tissues specimens (4- $\mu$ m-thick) were fixed in neutral buffered formalin and embedded in paraffin wax. Tissue sections were placed on charged glass slides, and then were subjected to deparaffinization in xylene and rehydration in a graded series of ethanol. Antigen retrieval was performed using citrate buffer at $\mathrm{pH}$ 6.0. Specimens were incubated with the antibodies against CAFs markers, and CD163 overnight at $4^{\circ} \mathrm{C}$ and washed in PBS for $10 \mathrm{~min}$. Information on antibodies was listed in Table 1.

The streptavidin-peroxidase technique (SP-9001 Golden Bridge Int, Beijing, China) was used. An irrelevant rabbit anti-serum served as a negative control. The slides were stained with 3-3'diamino-benzidine solution followed by counterstaining with hematoxylin.

Table 1. The list of manufacturers, origins, clones, dilution concentrations and incubation time of the antibodies

\begin{tabular}{|c|c|c|c|c|c|}
\hline & Manufacturer & Origin & Clone & $\begin{array}{l}\text { Dilution } \\
\text { concentration }\end{array}$ & $\begin{array}{l}\text { Incubation } \\
\text { time }\end{array}$ \\
\hline $\begin{array}{l}\alpha-\operatorname{SMA}(\mathrm{ab} \\
7818)\end{array}$ & $\begin{array}{l}\text { Abcam,, } \\
\text { Cambridge, } \\
\text { MA, USA }\end{array}$ & Mouse & $\begin{array}{l}\text { Mono- } \\
\text { clone }\end{array}$ & $1 / 200$ & $\begin{array}{l}14 \text { hours } \\
\text { Temperature: } \\
4^{\circ} \mathrm{C}\end{array}$ \\
\hline $\begin{array}{l}\text { FAP } \\
(\text { ab53066) }\end{array}$ & $\begin{array}{l}\text { Abcam, } \\
\text { Cambridge, } \\
\text { MA, USA }\end{array}$ & Rabbit & $\begin{array}{l}\text { Poly- } \\
\text { clone }\end{array}$ & $1 / 100$ & $\begin{array}{l}14 \text { hours } \\
\text { Temperature: } \\
4^{\circ} \mathrm{C}\end{array}$ \\
\hline $\begin{array}{l}\text { CD163 } \\
\text { (ab87099) }\end{array}$ & $\begin{array}{l}\text { Abcam," } \\
\text { Cambridge, } \\
\text { MA, USA }\end{array}$ & Rabbit & $\begin{array}{l}\text { Poly- } \\
\text { clone }\end{array}$ & $1 / 100$ & $\begin{array}{l}14 \text { hours } \\
\text { Temperature: } \\
4^{\circ} \mathrm{C}\end{array}$ \\
\hline
\end{tabular}

\section{Evaluation of immunohistochemistry}

All the slides were evaluated by two independent pathologists (X.Y.X. and S.Q.) who were blinded to the clinicopathological characteristics with consensus. All immunohistochemical markers were assessed via light microscopy (Carl Zeiss microscopy $\mathrm{GmbH}$, Jena, Germany). The expression levels of each marker in cancer cells, stromal fibroblasts and macrophages were independently evaluated. Immunohistochemistry data were evaluated as "low" or "high" expression, regarding the rate of positive cells for each sample and each marker as previously described [11, 18-21]. 


\section{Statistical analysis}

All the data were analyzed by Statistical Package for the Social Sciences (SPSS Inc., Chicago, IL, USA) for Windows version 17.0. The correlation between a-SMA, FAP and CD163 and clinicopathological features was analyzed by the chi-square test. The association of the expression level of CD163 with the activation of CAFs was analyzed by the Spearman's rank correlation co-efficient. The disease-free survival (DFS) and disease-specific survival (DSS) were the designated end-points. DFS was defined as the time from surgery to any local, regional, or distant disease; diagnosis of a second cancer other than breast cancer; or death from any cause. DSS was defined as the time period usually begins at the time of diagnosis of breast cancer or at the start of treatment and ends at the time of death. Patients who died from causes other than breast cancer are not counted in this measurement. All outcome results were reported as of May 31, 2016. Survival curves were calculated using the Kaplan-Meier method, and compared by log-rank test. DFS and OS rate curves were calculated through the Kaplan-Meier method. Multivariate analyses were analyzed by the Cox regression model. The $p$ values $<0.05$ were considered statistically significant.

\section{Results}

\section{Expressions of CAFs and TAMs markers in cancer tissues with clinicopathological features in TNBC}

The relationship between expressions of CAFs and TAMs markers and clinicopathological features of TNBC patients were described in Table 2. The study included 278 women, ranging from 20 to 84 years of age (mean, 57.8 years). More than half of the patients presented positive with lymph node (LN) (55.8\%, $155 / 278$ ), and patients presented with lymphovascular invasion (LVI) were $37.8 \%(105 / 278)$. Most cancers were larger than $2 \mathrm{~cm}$. As for histologic grade, more than two-thirds $(68.0 \%, 189 / 278)$ of cases were grade 3 . Ductal breast cancer was the predominant type, accounting for $94.6 \%(263 / 278)$.

The expressions of CAFs and TAMs markers were found predominantly in stromal cells and slightly in cancer cells in TNBC tissues. In stromal fibroblasts adjacent to cancer nests, a-SMA was reflected as brown cytoplasmic staining, and FAP was expressed on the cell membrane and in the cytoplasm. CD163 was detected on the cell membrane or in the cytoplasm of the macrophages both in tumor nests and stroma. High and low expressions of the above-mentioned proteins, according to typical IHC staining patterns, in breast cancer tissues were shown in Figure 1. Of the 278 patients, high expressions of
a-SMA and FAP, were detected in 50.4\% (140/278) and $52.5 \%(146 / 278)$ patients of TNBC, respectively. The high expression rates of $a$-SMA $(59.4 \%, 101 / 170)$ and $\operatorname{FAP}(63.5 \%, 108 / 170)$ in patients with recurrence were significantly higher than that without recurrence $(P<0.001$, respectively). The expressions of CAFs markers were also significantly correlated to the histological grade and aggressive behaviors of TNBC. High expressions of TAMs marker CD163, observed around the tumor nests in 148 cases $(53.2 \%)$, was closely related to the aggressive behaviors, such as advanced TNM stage, nodal metastasis, LVI and so on.

\section{Association of Expressions of CAFs Markers with Infiltration of TAMs and Lymphatic Metastasis}

The relationship between expressions of CAFs markers and CD163 were counted and has been described in Table 3. It was noted that expressions of CAFs markers in the 278 TNBC tissues were $50.4 \%$ for a-SMA and $52.5 \%$ for FAP. High infiltration of TAMs in neoplastic cells was also found in more than half cases. In overall patients, high infiltration of CAFs (a-SMA and FAP as markers) was significantly correlated with high infiltration of TAMs surrounding the cancer nests in TNBC tissues. As for recurrent cases, we found almost the similar results. In the same samples, we also found that high activation of CAFs was significantly correlated with lymphatic metastasis (Table 4).

\section{Combined High Activation of CAFs with High Infiltration of TAMs was Associated with Poor Prognosis for TNBC patients}

The follow-up time ranged from 8 to 130 months (median 87 months) and ended at May 31, 2016. When the follow-up was over, 197 patients were alive, including 90 cases without and 107 cases with recurrence, while the remaining 81 cases were dead (12 died of other accompanied diseases and 69 died due to tumor relapse).

Survival curves were analyzed by the Kaplan-Meier method and compared using the log-rank test. The mean survival time was $69.42 \pm 19.68$ months for all cases, and the 5-year DFS and DSS were $79.0 \%$ and $83.1 \%$, respectively. Compared with those with low expressions of CAFs markers, patients with high expressions had a disadvantageous DFS and DSS $(P<0.001)$. Multivariate Cox analysis showed that elevated activation of CAFs, high TAMs infiltration and lymphatic metastasis were independent worse prognostic factors for DFS and OS rates in TNBC patients $(P<0.05)$ (Fig. 2A-B, Table 5). 
Table 2. The relationship between expressions of CAFs and TAMs markers, and clinicopathologic features of TNBC patients

\begin{tabular}{|c|c|c|c|c|c|c|c|c|c|c|}
\hline & $\mathbf{N}$ & High a-SMA (\%) & $x^{2}$-value & $P$ & High FAP (\%) & $\chi^{2}$-value & $P$ & High CD163 (\%) & $x^{2}$-value & $P$ \\
\hline Age(yr) & & & 1.550 & 0.213 & & 0.115 & 0.735 & & 1.899 & 0.168 \\
\hline$\leq 40$ & 87 & $39(44.8)$ & & & $47(54.0)$ & & & $41(47.1)$ & & \\
\hline$>40$ & 191 & $101(52.9)$ & & & $99(51.8)$ & & & $107(56.0)$ & & \\
\hline Menstrual status & & & 1.700 & 0.192 & & 1.283 & 0.515 & & 2.545 & 0.111 \\
\hline Pre-menopause & 127 & $58(45.7)$ & & & $62(50.4)$ & & & $61(48.0)$ & & \\
\hline Post-menopause & 151 & $82(54.3)$ & & & $84(55.6)$ & & & $87(57.6)$ & & \\
\hline Tumor size(cm) & & & 5.800 & 0.016 & & 6.543 & 0.011 & & 4.621 & 0.032 \\
\hline$\leq 2$ & 71 & $27(38.0)$ & & & $28(39.4)$ & & & $30(42.3)$ & & \\
\hline$>2$ & 207 & $113(54.6)$ & & & $118(57.0)$ & & & $118(57.0)$ & & \\
\hline Histological grade & & & 5.143 & 0.023 & & 6.289 & 0.012 & & 5.843 & 0.016 \\
\hline $1 / 2$ & 89 & $36(40.4)$ & & & 37 (41.6) & & & $38(42.7)$ & & \\
\hline 3 & 189 & $104(55.0)$ & & & $109(57.7)$ & & & $110(58.2)$ & & \\
\hline Histological type & & & 0.087 & 0.769 & & 0.004 & 0.948 & & 0.275 & 0.600 \\
\hline Ductal & 263 & $133(50.6)$ & & & $138(52.5)$ & & & 141 (53.6) & & \\
\hline Lobular & 15 & 7 (46.7) & & & $8(53.3)$ & & & $7(46.7)$ & & \\
\hline LNS & & & 9.771 & 0.002 & & 12.460 & $<0.001$ & & 10.646 & 0.001 \\
\hline Negative & 123 & $49(39.8)$ & & & $50(40.7)$ & & & $52(42.3)$ & & \\
\hline Positive & 155 & $91(58.7)$ & & & $96(61.9)$ & & & $96(61.9)$ & & \\
\hline Recurrence & & & 14.343 & $<0.001$ & & 21.277 & $<0.001$ & & 14.606 & $<0.001$ \\
\hline Absent & 108 & $39(36.1)$ & & & $38(35.2)$ & & & $42(38.9)$ & & \\
\hline Present & 170 & $101(59.4)$ & & & 108 (63.5) & & & $106(62.4)$ & & \\
\hline LVI & & & 6.720 & 0.010 & & 5.962 & 0.015 & & 6.271 & 0.012 \\
\hline Absent & 173 & $77(44.5)$ & & & $81(46.9)$ & & & $82(47.3)$ & & \\
\hline Present & 105 & $63(60.0)$ & & & $65(61.9)$ & & & $66(62.9)$ & & \\
\hline
\end{tabular}

LNS: lymph node status, LVI: lymphatic vessel invasion

Table 3. The association of expressions of CAFs markers with infiltration of TAMs in overall and recurrent patients with TNBC

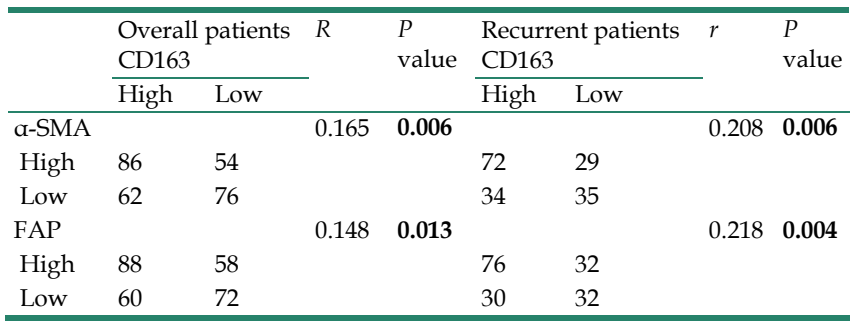

Table 4. The association of expressions of CAFs markers with lymphatic metastasis in overall and recurrent patients with TNBC

\begin{tabular}{|c|c|c|c|c|c|c|c|c|}
\hline & \multicolumn{2}{|c|}{$\begin{array}{l}\text { Overall patients } \\
\text { Lymph node status }\end{array}$} & \multirow[t]{2}{*}{$r$} & \multirow[t]{2}{*}{$\begin{array}{l}P \\
\text { value }\end{array}$} & \multicolumn{2}{|c|}{$\begin{array}{l}\text { Recurrent patients } \\
\text { Lymph node status }\end{array}$} & \multirow[t]{2}{*}{$r$} & \multirow[t]{2}{*}{$\begin{array}{l}P \\
\text { value }\end{array}$} \\
\hline & Positive & Negative & & & Positive & Negative & & \\
\hline a-SMA & & & 0.187 & 0.002 & & & 0.344 & $<0.001$ \\
\hline High & 91 & 49 & & & 78 & 23 & & \\
\hline Low & 64 & 74 & & & 30 & 39 & & \\
\hline FAP & & & 0.183 & 0.002 & & & 0.315 & $<0.001$ \\
\hline High & 93 & 53 & & & 81 & 27 & & \\
\hline Low & 62 & 70 & & & 27 & 35 & & \\
\hline
\end{tabular}

\section{Discussion}

In this study, we selected a cohort of 278 patients in order to analyze whether CAFs correlated with TAMs infiltration, lymphatic metastasis and could predict outcome for TNBC patients. The results of the present study supported the speculation that high activation of CAFs correlates with infiltration of TAMs, lymph node metastasis and contributes to poor prognosis in TNBC. The results also demonstrated that age (45.6 vs. $54.4 \%$ ), histological type and menopausal status ( 54.4 vs. $45.6 \%$ ) did not significantly affect the activation of CAFs and infiltration of TAMs. In the present study, we found that patients with aggressive features such as lymph node metastasis, large size, and high histological grade possessed with high expression of CAFs markers and elevated of TAMs infiltration. In addition, CAFs markers significantly correlated with CD163 expression and lymph node metastasis.

In the current study, we also manifested that CAFs markers significantly correlated with CD163 expression and lymph node metastasis, especially for those with recurrence. The combination of CAFs and TAMs within a tumor site has been reported to be a potential prognostic factor in multiple solid cancers, such as oral cancer, neuroblastoma and colorectal cancer patients [12-14]. In the present study, Multivariate Cox showed that activation of CAFs, high TAMs infiltration and lymphatic metastasis were independent worse prognostic factors in TNBC patients.

In breast cancer, CAFs are key players in the tumor microenvironment since they not only promote tumorigenesis, cancer evolution and therapeutic resistance, and targeting CAFs would provide a novel strategy for anti-cancer treatment [7, 22]. Recently, accumulating researches have focused on the role of CAFs in TNBC. Increased collagen expression in tumors is associated with increased risk of metastasis, and TNBC has the highest propensity to develop distant metastases when there is evidence of central 
fibrosis. CAFs can regulate TGF- $\beta$ ligands to promote accumulation of fibrosis and cancer progression. By targeting the CAFs with Pirfenidone (PFD) in combination with doxorubicin, could inhibit tumor growth and lung metastasis synergistically [23]. Hu C et al. Shown that regulating CAFs with losartan-loaded injectable peptide hydrogel could potentiate the effect of chemotherapy in inhibiting growth and lung metastasis TNBC [24]. The study conducted by Wang $\mathrm{M}$ et al. demonstrated that CAFs autophagy can enhance TNBC cell migration, invasion, and proliferation, and induce EMT process through the Wnt/ $\beta$-catenin signal pathway [25].
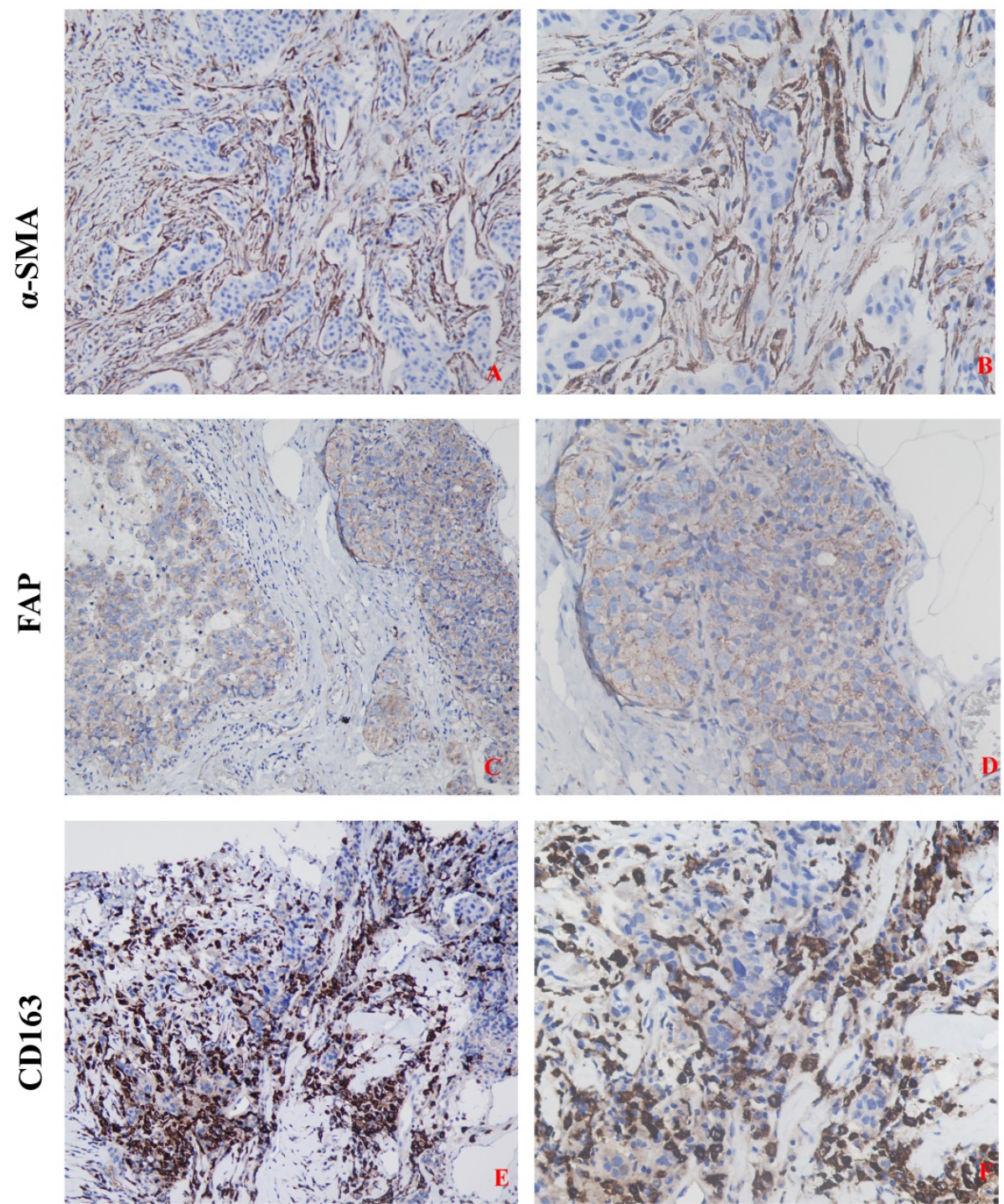

Figure 1. Detection of CAFs activation ( $\alpha-S M A$ and FAP) and TAMs infiltration ( marker CD163) in TNBC tissue by IHC. High CAFs activation and elevated infiltration of TAMs was seen in more aggressive cancer. A-B: high expression of Q-SMA ( A: $200 \times$; B: $400 \times$ ) and C-D: high expression of FAP ( C: $200 \times$; D: 400 $\times$ ) ; E-F: Strong immunoreactivity of CD163 ( E: $200 \times$; F: $400 \times$ ).

Table 5. Multivariate analysis of significant prognostic factors for DFS and DSS for patients with TNBC

\begin{tabular}{|c|c|c|c|c|c|c|}
\hline \multirow[t]{2}{*}{ Variables } & \multicolumn{3}{|l|}{ DFS } & \multicolumn{3}{|l|}{ DSS } \\
\hline & HR & $95 \% \mathrm{CI}$ & $P$-value & HR & $95 \% \mathrm{CI}$ & $P$-value \\
\hline Tumor size, $\mathrm{cm}(\leq 2 \mathrm{vs}>2)$ & 1.992 & $1.146-3.464$ & 0.047 & 1.818 & $0.829-3.985$ & 0.074 \\
\hline LNS (Negative vs Positive) & 2.147 & $1.229-3.749$ & 0.007 & 2.247 & $1.355-3.725$ & 0.005 \\
\hline LVI (Absent vs Present) & 2.089 & $1.309-3.333$ & 0.021 & 1.913 & $1.244-2.942$ & 0.040 \\
\hline Histological grade (1/2 vs 3 ) & 1.616 & $1.042-2.505$ & 0.053 & 1.850 & $1.177-2.907$ & 0.062 \\
\hline a-SMA expression (Low vs High) & 2.477 & $1.218-5.037$ & 0.010 & 2.831 & $1.373-5.837$ & 0.003 \\
\hline FAP expression (Low vs High) & 2.501 & $1.532-4.082$ & 0.003 & 3.044 & $1.799-5.151$ & $<0.001$ \\
\hline TAM infiltration (Low vs High) & 3.225 & $1.253-8.300$ & $<0.001$ & 3.113 & $1.489-6.509$ & 0.009 \\
\hline
\end{tabular}



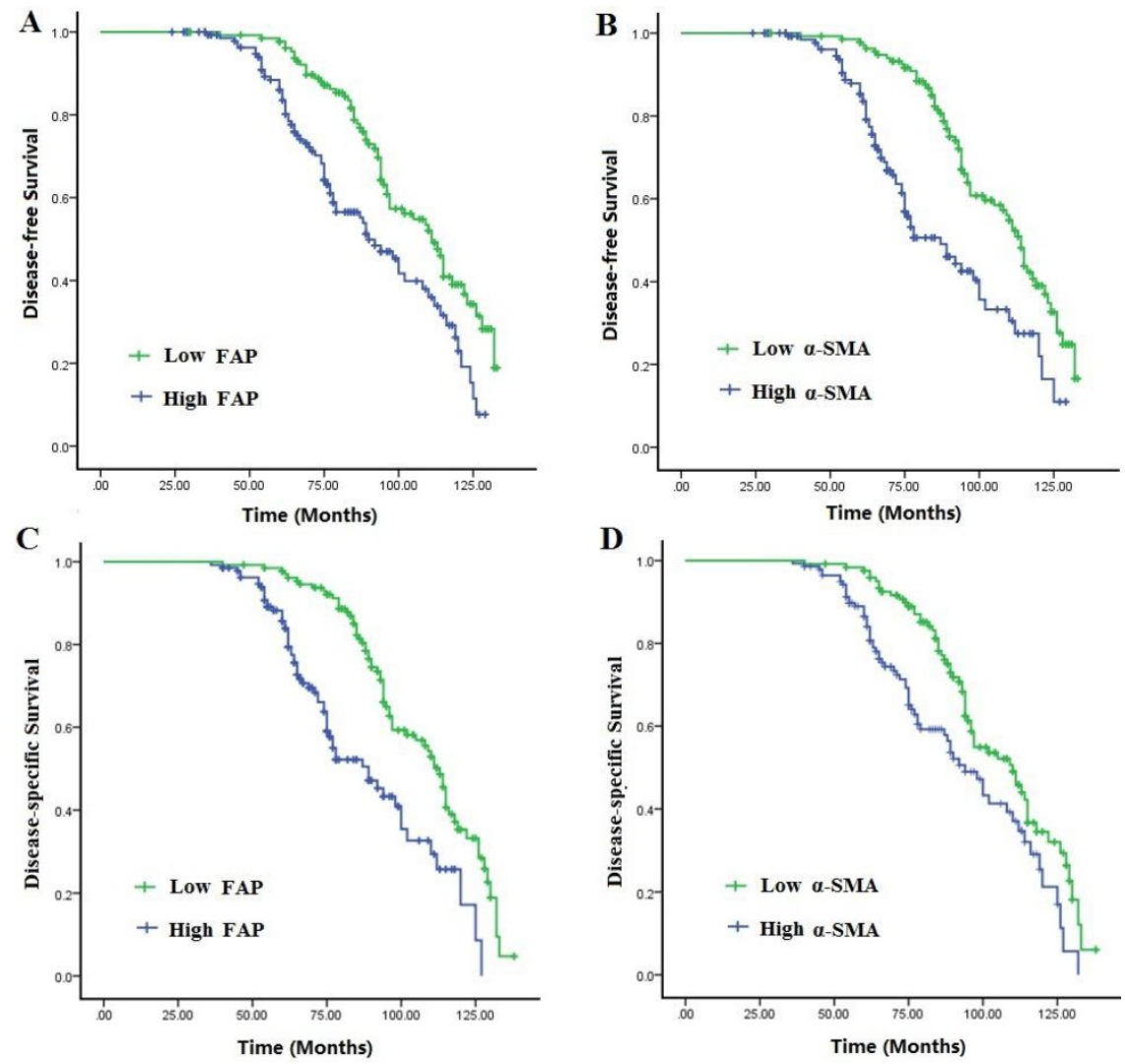

Figure 2. Disease-free survival (DFS) and disease-specific survival (DSS) of the 278 patients with TNBC in relation to $\alpha$-SMA and FAP expressions in CAFs. Patients with high activation of CAFs had a worse DFS and DSS than those with low activation.

In this retrospective study, our results suggest that CAFs were correlated with TAMs infiltration in patients with TNBC. High expressions of a-SMA and FAP were correlated with elevated expression of CD163, and CAFs might play an important role in shaping the tumor immunosuppressive microenvironment by regulating protumoral phenotype of TAMs. However, the exact mechanism of CAFs polarize macrophage is not yet clarified in TNBC. The research conducted by Kumar $\mathrm{V}$ et al. has demonstrated that CAFs are major sources of chemokines that can recruit and induce PMN-MDSC infiltration to neutralize the anti-tumor effect of CSF1 receptor blockade to eliminate TAMs [26]. Crosstalk between stromal components and TNBC cells could enhance TNBC tumor growth and metastatic extravasation and colonization, and the IL-8-CXCR1/2 signaling acts as a key regulator orchestrating TNBC metastatic breast cancer via TNBC crosstalk with CAFs and TAMs [27]. The findings of Tashireva LA et al. has shown the heterogeneous distribution of fibroblasts and macrophages in breast tumor microenvironment and its close relation to the intratumoral morphological diversity of $\mathrm{BC}$ and contribution to lymph node metastasis [28]. In breast cancer, CAFs could promote an immunosuppressive and growth-promoting microenvironment through the induction and accumulation of protumoral macrophages via secretion of Chitinase 3-like 1 [29]. In turn, TAMs can affect collagenous matrix remodeling through their regulation of collagen production by CAFs and induce myofibroblast differentiation [30, 31]. Therefore, due to the enrichment in reactive CAFs and M2-like macrophages in TNBC stromal, the crosstalk between CAFs and TAMs might be a potentially novel target in ameliorating the pro-tumorigenic microenvironment.

Our data show that CAFs activation correlated with TAMs infiltration and lymph node metastasis, and there may be exist a fascinating loop among CAFs, TAMs and cancer cells, in which the interaction between stromal and cancer cells allows for the establishment of a pro-inflammatory microenvironment to promote cancer progress. In keeping with our findings and these reports, the combination of TAMs and CAFs could be a potential prognostic factor and also a potential therapeutic target in TNBC.

\section{Acknowledgments}

This work was supported by grants from the National Natural Science Foundation of China (Grant Nos. 81201909, 81572338 and 81672380), Nanjing Medical Science and Technology Development 
program (Nos. YKK12072 and YKK15061). This work was also a $\mathrm{C}$ class sponsored project of Jiangsu provincial Six Talent Peaks (WSN-078).

\section{Competing Interests}

The authors have declared that no competing interest exists.

\section{References}

1. Chen W, Zheng R, Baade PD, et al. Cancer statistics in China, 2015. CA Cancer J Clin. 2016 ;66(2):115-32.

2. Badve S, Dabbs DJ, Schnitt SJ, et al. Basal-like and triple-negative breast cancers: a critical review with an emphasis on the implications for pathologists and oncologists. Mod Pathol. 2011;24(2):157-67.

3. Rakha EA, Ellis IO. Triple-negative/basal-like breast cancer: review. Pathology. 2009;41(1):40-7

4. Bianchini G, Balko JM, Mayer IA, et al. Triple-negative breast cancer: challenges and opportunities of a heterogeneous disease. Nat Rev Clin Oncol. 2016 ; 13(11):674-690.

5. Qiu J, Xue X, Hu C, et al. Comparison of Clinicopathological Features and Prognosis in Triple-Negative and Non-Triple Negative Breast Cancer. J Cancer. 2016;7(2):167-73.

6. Luo A, Wu F, Han R, et al. Clinicopathological features and prognostic evaluation of bone metastasis in triple-negative breast cancer. J Cancer Res Ther. 2017;13(5):778-784.

7. Luo H, Tu G, Liu Z, et al. Cancer-associated fibroblasts: a multifaceted driver of breast cancer progression. Cancer Lett. 2015;361(2):155-63.

8. Jung YY, Kim HM, Koo JS. The role of cancer-associated fibroblasts in breast cancer pathobiology. Histol Histopathol. 2016 ;31(4):371-8

9. Soon PS, Kim E, Pon CK, et al. Breast cancer-associated fibroblasts induce epithelial-to-mesenchymal transition in breast cancer cells. Endocr Relat Cancer. 2013;20(1):1-12

10. Yuan ZY, Luo RZ, Peng RJ, et al. High infiltration of tumor-associated macrophages in triple-negative breast cancer is associated with a higher risk of distant metastasis. Onco Targets Ther. 2014 ;7:1475-80.

11. Zhang WJ, Wang XH, Gao ST, et al. Tumor-associated macrophages correlate with phenomenon of epithelial-mesenchymal transition and contribute to poor prognosis in triple-negative breast cancer patients. J Surg Res. 2018;222:93-101.

12. Fujii $\mathrm{N}$, Shomori $\mathrm{K}$, Shiomi $\mathrm{T}$, et al. Cancer-associated fibroblasts and CD163-positive macrophages in oral squamous cell carcinoma: their clinicopathological and prognostic significance. J Oral Pathol Med. 2012;41(6):444-51.

13. Herrera M, Herrera A, Domínguez G, et al. Cancer-associated fibroblast and M2 macrophage markers together predict outcome in colorectal cancer patients. Cancer Sci. $2013 ; 104(4): 437-44$.

14. Hashimoto O, Yoshida M, Koma Y, et al. Collaboration of cancer-associated fibroblasts and tumour-associated macrophages for neuroblastoma development. J Pathol. 2016 ;240(2):211-23.

15. Comito G, Giannoni E, Segura CP, et al. Cancer-associated fibroblasts and M2-polarized macrophages synergize during prostate carcinoma progression. Oncogene. $2014 ; 33(19): 2423-31$.

16. Zhang A, Qian Y, Ye Z, et al. Cancer-associated fibroblasts promote M2 polarization of macrophages in pancreatic ductal adenocarcinoma. Cancer Med. $2017 ; 6(2): 463-470$.

17. Edge SB, Byrd DR, Compton CC, et al. AJCC cancer staging manual (7th ed). New York, NY: Springer; 2010, 347-376

18. Chen $Y$, Zou L, Zhang $Y$, et al. Transforming growth factor- $\beta 1$ and a-smooth muscle actin in stromal fibroblasts are associated with a poor prognosis in patients with clinical stage I-IIIA nonsmall cell lung cancer after curative resection. Tumour Biol. $2014 ; 35(7): 6707-13$.

19. Yamashita M, Ogawa T, Zhang X, et al. Role of stromal myofibroblasts in invasive breast cancer: stromal expression of alpha-smooth muscle actin correlates with worse clinical outcome. Breast Cancer. 2012;19(2):170-6.

20. Tchou J, Zhang PJ, Bi Y, et al. Fibroblast activation protein expression by stromal cells and tumor-associated macrophages in human breast cancer. Hum Pathol. 2013 ;44(11):2549-57.

21. Patsouras D, Papaxoinis K, Kostakis A, et al. Fibroblast activation protein and its prognostic significance in correlation with vascular endothelial growth factor in pancreatic adenocarcinoma. Mol Med Rep. 2015;11(6):4585-90.

22. Su S, Chen J, Yao H, et al. CD10+GPR77+ Cancer-Associated Fibroblasts Promote Cancer Formation and Chemoresistance by Sustaining Cancer Stemness. Cell. $2018 ; 172(4): 841-856$.

23. Takai K, Le A, Weaver VM, et al. Targeting the cancer-associated fibroblasts as a treatment in triple-negative breast cancer. Oncotarget. 2016 ;7(50):82889-82901.

24. Hu C, Liu X, Ran W, et al. Regulating cancer associated fibroblasts with losartan-loaded injectable peptide hydrogel to potentiate chemotherapy in inhibiting growth and lung metastasis of triple negative breast cancer. Biomaterials. $2017 ; 144: 60-72$
25. Wang M, Zhang J, Huang Y, et al. Cancer-Associated Fibroblasts Autophagy Enhances Progression of Triple-Negative Breast Cancer Cells. Med Sci Monit. $2017 ; 23: 3904-3912$

26. Kumar V, Donthireddy L, Marvel D, et al. Cancer-Associated Fibroblasts Neutralize the Anti-tumor Effect of CSF1 Receptor Blockade by Inducing PMN-MDSC Infiltration of Tumors. Cancer Cell. 2017 ;32(5):654-668.

27. Jin K, Pandey NB, Popel AS. Crosstalk between stromal components and tumor cells of TNBC via secreted factors enhances tumor growth and metastasis. Oncotarget. $2017 ; 8(36): 60210-60222$.

28. Tashireva LA, Denisov EV, Gerashchenko TS, et al. Intratumoral heterogeneity of macrophages and fibroblasts in breast cancer is associated with the morphological diversity of tumor cells and contributes to lymph node metastasis. Immunobiology. 2017 ;222(4):631-640.

29. Cohen N, Shani O, Raz Y, et al. Fibroblasts drive an immunosuppressive and growth-promoting microenvironment in breast cancer via secretion of Chitinase 3-like 1. Oncogene. 2017 ;36(31):4457-4468.

30. Afik R, Zigmond E, Vugman $\mathrm{M}$, et al. Tumor macrophages are pivotal constructors of tumor collagenous matrix. J Exp Med. 2016 ;213(11):2315-2331.

31. Nie Y, Chen J, Huang D, et al. Tumor-Associated Macrophages Promote Malignant Progression of Breast Phyllodes Tumors by Inducing Myofibroblast Differentiation. Cancer Res. 2017 ;77(13):3605-3618. 